\title{
Editorial Comment on: MicroRNA-593-3p Expression in Peritoneal Lavage Fluid as a Prognostic Marker for Pancreatic Cancer Patients Undergoing Staging Laparoscopy
}

\author{
Takehiko Yokobori, MD, $\mathbf{P h D}^{1,2}$ \\ ${ }^{1}$ Department of General Surgical Science, Graduate School of Medicine, Gunma University, Maebashi, Japan; ${ }^{2}$ Research \\ Program for Omics-Based Medical Science, Division of Integrated Oncology Research, Gunma University Initiative for \\ Advanced Research (GIAR), Maebashi, Japan
}

Pancreatic cancer is a highly aggressive malignancy with a high mortality rate. ${ }^{1}$ Despite improvements in the surgical management of pancreatic cancer, the prognosis of patients with unresectable diseases such as peritoneal dissemination remains poor. ${ }^{2}$ Therefore, reliable biomarkers for detecting invisible peritoneal dissemination can improve decision-making regarding treatment. Suitable therapeutic strategies are based on pancreatic cancer staging by preoperative imaging modalities and staging laparoscopy.

MicroRNAs (miRs) are small, noncoding RNAs of approximately 22 nucleotides, which partially bind to the 3-untranslated region (3-UTR) of target mRNAs, leading to mRNA degradation and/or translational repression. ${ }^{3,4}$ MiRs have attracted attention, not only as gene expression-regulatory factors, but also as biomarkers in several body fluids, depending on disease-specific miRNA expression profiles. ${ }^{5}$ Rosenfeld et al. reported the interesting characteristics of miRNA expression profiles, which were useful for identifying the tissue origin of cancers of unknown primary origin. ${ }^{6}$ These findings strongly suggested the potential of miRs in body fluids as effective diseasespecific biomarkers.

(C) Society of Surgical Oncology 2020

First Received: 17 November 2020

Accepted: 18 November 2020;

Published Online: 28 November 2020

T. Yokobori, MD, $\mathrm{PhD}$

e-mail: bori45@gunma-u.ac.jp
Researchers have investigated the expression of miRs in peritoneal fluids as biomarker candidates in patients with gastric, endometrial, and pancreatic cancer. ${ }^{7-11}$ In this issue of Annals of Surgical Oncology, Hata et al. report the ability of miR-593-3p expression in peritoneal lavage to predict poor prognosis in pancreatic cancer patients. ${ }^{12}$ First, the authors discovered upregulated miR-593-3p in peritoneal lavage fluid of pancreatic cancer patients who tested positive on cytology via miRNA array analysis. Moreover, they validated the high expression levels of miR-593-3p in peritoneal lavage and clarified its role as an independent prognostic factor in patients after pancreatectomy. Droplet digital PCR methods with high sensitivity and quantitativity were used instead of existing real-time PCR methods. Based on these findings, the miR biomarker is useful for diagnosing occult peritoneal dissemination in pancreatic cancer patients.

Hata's research was a retrospective observational study, and the following issues should be considered in the future: First, was the miR-593-3p in the peritoneal cavity secreted by cancer cells, host cells, or both? Second, was the miR593-3p in the peritoneal lavage a progressive and prognostic biomarker, or did it have a significant functional role in pancreatic cancer progression? Third, can the miR-593$3 p$ in the peritoneal cavity be a new therapeutic target for pancreatic cancer patients? Therefore, miR-593-3p evaluation in peritoneal lavage may enable clinicians to accurately identify patients with residual invisible lesions and recurrence after surgery as well as determine more appropriate adjuvant chemotherapy indications. Further prospective studies with large cohorts and functional 
analysis of miR-593-3p will be conducted to validate the significance of miR-593-3p as a biomarker and target candidate in pancreatic cancer patients.

DISCLOSURES The author declares no conflicts of interest.

\section{REFERENCES}

1. Strobel O, Neoptolemos J, Jager D, Buchler MW. Optimizing the outcomes of pancreatic cancer surgery. Nat Rev Clin Oncol. 2019;16(1):11-26.

2. Nevala-Plagemann C, Hidalgo M, Garrido-Laguna I. From stateof-the-art treatments to novel therapies for advanced-stage pancreatic cancer. Nat Rev Clin Oncol. 2020;17(2):108-23.

3. Mardin WA, Mees ST. MicroRNAs: novel diagnostic and therapeutic tools for pancreatic ductal adenocarcinoma? Ann Surg Oncol. 2009;16(11):3183-9.

4. Lin S, Gregory RI. MicroRNA biogenesis pathways in cancer. Nat Rev Cancer. 2015;15(6):321-33.

5. Kosaka N, Iguchi H, Ochiya T. Circulating microRNA in body fluid: a new potential biomarker for cancer diagnosis and prognosis. Cancer Sci. 2010;101(10):2087-92.

6. Rosenfeld N, Aharonov R, Meiri E, et al. MicroRNAs accurately identify cancer tissue origin. Nat Biotechnol. 2008;26(4):462-9.
7. Tokuhisa M, Ichikawa Y, Kosaka N, et al. Exosomal miRNAs from peritoneum lavage fluid as potential prognostic biomarkers of peritoneal metastasis in gastric cancer. PLOS ONE. 2015;10(7):e0130472.

8. Roman-Canal B, Moiola CP, Gatius S, et al. EV-associated miRNAs from peritoneal lavage are a source of biomarkers in endometrial cancer. Cancers. 2019;11(6):839.

9. Roman-Canal B, Tarragona J, Moiola CP, et al. EV-associated miRNAs from peritoneal lavage as potential diagnostic biomarkers in colorectal cancer. J Transl Med. 2019;17(1):208.

10. Kubo H, Hiroshima Y, Mori R, et al. MiR-194-5p in pancreatic ductal adenocarcinoma peritoneal washings is associated with peritoneal recurrence and overall survival in peritoneal cytologynegative patients. Ann Surg Oncol. 2019;26(13):4506-14.

11. Ohzawa H, Saito A, Kumagai Y, et al. Reduced expression of exosomal miR29s in peritoneal fluid is a useful predictor of peritoneal recurrence after curative resection of gastric cancer with serosal involvement. Oncol Rep. 2020;43(4):1081-8.

12. Hata T, Mizuma M, Masuda K, et al. MicroRNA-593-3p expression in peritoneal lavage fluid as a prognostic marker for pancreatic cancer patients undergoing staging laparoscopy. Ann Surg Oncol. 2020. https://doi.org/10.1245/s10434-020-09440-3.

Publisher's Note Springer Nature remains neutral with regard to jurisdictional claims in published maps and institutional affiliations. 